\title{
Familiar size and linear perspective as distance cues in stereoscopic depth constancy
}

\author{
ANN O'LEARY \\ University of Pennsylvania, Philadelphia, Pennsylvania 19104 \\ and \\ HANS WALLACH \\ Swarthmore College, Swarthmore, Pennsylvania 19081
}

\begin{abstract}
Both the image size of a familiar object and linear perspective operate as distance cues in stereoscopic depth constancy. This was shown by separating their effects from the effect of the oculomotor cues by creating cue conflicts between either the familiar size cue or linear perspective, on the one hand, and accommodation and convergence, on the other. In the case of familiar size, this cue was used deceptively. In the case of linear perspective, spectacles caused nonveridical oculomotor adjustments.
\end{abstract}

In their chapter on stereoscopic depth constancy, Ono and Comerford (1977) discussed the question of whether only the oculomotor adjustments of convergence and accommodation calibrate retinal disparity in such a way that constancy results, or if other distance cues also can provide the distance information necessary for stereoscopic depth perception. Our experiments deal with this question.

In order for a binocularly given depth interval to be perceived correctly, the disparity inherent in the differing projections on the two retinas must be evaluated on the basis of the absolute distance of the depth interval from the eyes. This is because a fixed depth interval produces a retinal disparity that decreases with increasing observation distance. More precisely, disparity is inversely proportional to the square of the observation distance. ${ }^{1}$ It is known that stereoscopic depth perception compensates for this decrease in disparity with observation distance up to a distance of $200 \mathrm{~cm}$. In this compensation process, which is called constancy of stereoscopic depth, perceived depth is roughly equivalent to disparity times the square of observation distance as represented by the available distance cues. While it has been conclusively shown that the oculomotor cues for observation distance operate in depth perception (Wallach \& Zuckerman, 1972), the possibility that other distance cues may also be used has not been investigated in detail. Our first experiment explored whether distance information produced by the size of a familiar object could serve this function.

This work was supported by Grant BNS75-19095 A01 from the National Science Foundation to Swarthmore College, Hans Wallach, principal investigator. We are most grateful to Martha Teghtsoonian for her help in writing this report.

\section{FAMILIAR SIZE}

That the angular sizes of objects known to be constant in size, such as playing cards, dollar bills, and matchbooks, can serve as cues for observation distance is well known (e.g., Experiment 5 in Wallach, Frey, $\&$ Bode, 1972). Ordinarily, the perceived size of an object is derived from its angular size times its registered distance, which is its observation distance as represented by the available distance cues. When a familiar object serves as a distance cue, both angular size and perceived size are given, with the latter based on memory. Perceived distance is derived from the proportion of perceived size to angular size. In our experiment, two dollar bills, one normal-sized and one a smaller reproduction whose linear dimensions were .72 of normal size, were presented at the same distance from the subject. Each was paired with a small white disk to produce a small depth interval. If the sizes of these bills were operating as distance cues, the distance of the smaller bill would be registered as 1/.72 times larger than the distance of the larger bill, and if the projective sizes of familiar objects can serve as distance cues in stereoscopic depth constancy, the depth connected with the smaller bill should appear to be greater. Of course, convergence and accommodation of the eyes would operate as distance cues also, but they would represent the objective distances, which were equal. Another veridical distance cue that could be present in our experiment was head movement parallax.

\section{Method}

There were 12 subjects. Prior to participating in the experiment, they were tested for stereoscopic depth perception. Two stereoscopic displays were used. In one, three vertical lines were seen by each eye, with disparities so arranged that the middle 
one appeared to be located behind the other two. The other was a reproduction of a random-dot pattern stereogram taken from a paper by Julesz, of the kind in which a small square appeared in front of a larger one. All of the prospective subjects passed. this test.

Two displays were constructed. Each consisted of one of the bills, mounted on black construction paper with a small white disk arranged $1.0 \mathrm{~cm}$ in front of and above the bill so that its edge slightly overlapped that of the bill when viewed by the subject. The dimensions of the black paper were transposed to correspond to those of the bills. The disk sizes were similarly transposed for half of our 12 subjects; the larger disk measured $1.7 \mathrm{~cm}$ across. For 3 of the remaining 6 subjects, both disks were of the larger size; for the other three they were of the smaller size.

Both displays were located $75 \mathrm{~cm}$ from the subject's eyes and $70 \mathrm{~cm}$ apart and were alternately illuminated with small beams of light in an otherwise dark room. The subject had to give estimates of the apparent depth between the disk and the bill by adjusting a set of outside calipers to represent the perceived depth. Because there was no illumination on the calipers, the subjects used their sense of touch only. The subject's chin rested on a bar, so that he or she could pivot the head toward each display.

Each subject made three caliper adjustments for each of the displays. The displays were presented alternately, with the order of presentation reversed for half the subjects in each condition. After each pair of presentations, the experimenter asked the subject to close his eyes and pretended to alter the display. The instructions were first to inspect the bill until he received a signal and then to pay attention to the interval between the disk and the bill and to adjust the calipers. The initial inspection of the bill lasted $10 \mathrm{sec}$. The calipers were always given to the subject in the closed position. After the adjustment had been made, the experimenter turned off the display light and, using a flashlight, marked the caliper position on paper to be measured later.

\section{Results and Discussion}

The three caliper adjustments that a subject made in connection with each of the two displays were averaged, and the two results became his two depth estimation scores. For all 12 subjects, the mean estimate of the depth between the normal bill and its disk was $1.15 \mathrm{~cm}$, not very different from the objective depth of $1.0 \mathrm{~cm}$. All depth estimate scores that were obtained in connection with the small bill reproduction were larger than those given for the normal bill display, and for only 2 of the 12 subjects did the two sets of caliper adjustments, the three for the larger and the three for the smaller bill, overlap slightly. The mean depth estimate obtained in connection with the small bill was $1.71 \mathrm{~cm}$ and the difference between this mean and the mean depth estimate for the normal bill of $1.15 \mathrm{~cm}$ was significant [t(11) $=6.37, \mathrm{p}<.001]$. The mean difference between depth estimates of $.61 \mathrm{~cm}$ obtained for the transposed disks condition and the mean difference between depth estimates of $.52 \mathrm{~cm}$ obtained for the equal disk condition were not significantly different from each other. Each of these differences, however, was significant by itself $[t(5)$ $=4.21, \mathrm{p}<.01$, and $\mathrm{t}(5)=5.27, \mathrm{p}<.01$, respectively].

The mean depth estimate connected with the small bill was not as large as it would have been if the size of that bill had been fully effective as a distance cue.
In that case, the registered distance of the depth interval connected with the small bill would have been $1 / .72$, or 1.39 times larger than that of the normal bill, and the resulting perceived depth would have been larger than the depth connected with the normal bill by a factor of 1.15 times $(1.39)^{2}$, or $2.22 \mathrm{~cm}$. The obtained mean was $1.71 \mathrm{~cm}$ and the shortfall was significant $[t(11)=3.27, p<.01]$. Such a shortfall was to be expected because of the presence of convergence and accommodation, which represented the distance of the small bill correctly. Since the distance of the two bills from the subject's eyes was the same, the perceived depth based on these cues should have been the same. That the increase in depth caused by the small bill was about half of what it would have been had the size of the small bill been fully effective is most likely the result of this cue conflict. The highly significant difference in perceived depth connected with bills of different sizes indicates that familiar size is utilized as a distance cue in stereoscopic depth constancy.

The question may be raised as to whether the proportion of the image sizes of the bills, along with the papers on which they were mounted, served as cues for their different observation distances. The relative size cue, rather than the image size of a familiar object, may have been responsible for the larger depth we measured at the smaller bill. This question can be answered by comparing only the first depth estimates obtained from each subject. The mean depth estimate in connection with the normal bill was $.78 \mathrm{~cm}$ when the scores used were only of those subjects who saw the normal bill first; the corresponding mean for the small bill was $1.64 \mathrm{~cm}$. The difference of $.86 \mathrm{~cm}$ $[t(10)=3.45, p<.01]$ is even larger than the difference of $.56 \mathrm{~cm}$ between the means when the second estimates are included. There is no evidence that the proportion of image sizes had an effect in our experiment.

\section{LINEAR PERSPECTIVE}

When one attempts to show that a particular kind of distance cue can function in stereoscopic depth constancy, one faces the problem that accommodation and convergence are always present in stereoscopic vision, and that, therefore, correctly perceived depth can be attributed to their operation as distance cues. To demonstrate that a distance cue other than these oculomotor cues is effective in stereoscopic vision requires contrived experimental conditions in which that cue is placed in conflict with the oculomotor cues. If such conditions yield perceived depth that reflects an influence of the distance cue under consideration, its effectiveness is demonstrated. In the preceding experiment, a conflict between the familiar size cue and the oculomotor cues was created by using fa- 
miliar size deceptively. The registered distance of the small bill was larger than its physical distance, causing the associated depth interval to be perceived as larger than the depth interval caused by fully veridical distance cues.

In the present experiment, the oculomotor cues were given deceptively. ${ }^{2}$ The subject wore spectacles that altered accommodation and convergence. They consisted of -1.5 -diopter meniscus lenses which caused the eyes to focus for object distances with an accommodation larger by 1.5 diopters than the physical distance of the object would warrant. These lenses were combined with meniscus prisms that were oriented with bases temporal and were of a strength (5 prism diopters) that caused a change of convergence equivalent to the change in accommodation produced by the lenses. These spectacles caused oculomotor adjustments for distances that were shorter than the true object distances, thus producing registered distances that were too small. Because the spectacles cause changes in accommodation and convergence of constant dioptric value, they alter larger distances more than shorter distances. We used observation distances of 40 and $80 \mathrm{~cm}$, which the eyes, looking through the spectacles, viewed with oculomotor adjustments for 25 and $36.4 \mathrm{~cm}$.

\section{Method}

The depth interval estimated by the subject was formed by two vertical metal rods, $3.2 \mathrm{~mm}$ wide, mounted $21 \mathrm{~mm}$ apart on a small metal base. The plane of the rod pair always formed an angle of $60 \mathrm{deg}$ with the subject's frontal plane. The rod nearer to the subject was $50 \mathrm{~mm}$ high, and the more distant one $60 \mathrm{~mm}$. The rods were of different lengths to have the pair produce transverse disparity (Wallach \& Bacon, 1976). The longer rod was in the rear to prevent the size difference from providing a perspective cue for the depth order. The metal base was covered with 3-cm-wide strips of black construction paper that concealed the top of the base and the lower ends of the rods from the subject, since the latter would have provided perspective cues for the depth between the rods had they been visible. There were two such rod pairs for simultaneous comparison. The rod pair that was $40 \mathrm{~cm}$ distant from the subject's eyes was slightly to the left of his median plane, and the rod pair $80 \mathrm{~cm}$ distant slightly to the right. The visual angle between the rod pairs was $6.5 \mathrm{deg}$.

There were two viewing conditions in which the subject wore the glasses. In one of these conditions (GP), perspective cues were provided. In the other condition $(G)$, perspective cues were absent. In a third condition (NG), the subject wore no glasses and there were no perspective cues. In the two conditions ( $G$ and $N G$ ) in which perspective cues were absent, the metal blocks with the rod pairs were mounted on tall narrow stands $40 \mathrm{~cm}$ above the table top. In the GP condition, they rested on a platform, $80 \mathrm{~cm}$ long and $50 \mathrm{~cm}$ wide, that was $32.3 \mathrm{~cm}$ above the table top and covered with a checkered oilcloth, and provided the perspective cues. An attachment for a teeth mold was provided that kept the subject's head at the proper distance from the rod pairs. Its height was set so that the subject's eyes were $11 \mathrm{~cm}$ above the top of the platform, whose pattern was therefore clearly visible.

A subject gave his or her depth estimate by drawing on a small paper pad an oblique line that represented the slanting plane of the rod pair. He was permitted to look at the rod pair until he felt ready to give his slant estimate. Then he raised a finger and the experimenter handed him pad and pencil. In the NG condition, these slant estimates represented, of course, normal stereoscopic depth constancy. In the $G$ condition, the slant estimates represented an effect of the glasses, which caused, for example, the rod pair at the distance of $80 \mathrm{~cm}$ to be given with oculomotor adjustment for a distance of $36.4 \mathrm{~cm}$. If only oculomotor cues were effective in the $G$ condition, registered distance for the rod pair at $80 \mathrm{~cm}$ should have been diminished by the ratio of 80 to 36.4 , that is, by a factor of 2.2 , and perceived depth by the square of 2.2 . The perceived slant of the rod pair, however, cannot be expected to undergo a corresponding change. The glasses also have an effect on perceived size, including the spacing of the rods, and perceived slant depends on both perceived depth and perceived size. Because perceived size is proportional to registered distance and perceived depth is proportional to the square of registered distance, the change in slant can be expected to correspond to the first power of the change in registered distance. This means a reduction in depth by a factor of 2.2 in the case of the $80-\mathrm{cm}$ distance and by a factor of 1.6 for the $40-\mathrm{cm}$ distance.

In all conditions, the rod pair was presented first at the $80-\mathrm{cm}$ distance and then at the $40-\mathrm{cm}$ distance, and a slant estimate was obtained at each presentation. In the $G$ and the GP conditions, this sequence was repeated once, and subsequently slant estimates were obtained with two rod pairs simultaneously visible, one at each distance. (Eventually, the results showed that the slant estimates obtained at these second presentations or when rod pairs at both distances were simultaneously visible could not be used.) In the NG condition, only one estimate was obtained at each distance. There were two different orders of presentation: Either the $\mathbf{G}$ condition preceded the GP condition or this sequence was reversed. The NG condition always came after the $G$ and the GP conditions.

Twelve selected subjects, paid undergraduates, served in this experiment. The test for stereovision that was used to select subjects in Experiment 1 was made harder by adding two charts. Both showed two vertical lines for each eye with a disparity between them; in one, the lines were of equal length and in the other, one line was shorter. In the selection of the 12 subjects, six potential subjects were rejected.

\section{Results and Discussion}

The results listed in Table 1 will be used in three comparisons. (1) The means of the slant angle estimates that were given without the glasses in the NG condition will be compared with the actual slant angles to show that the technique of slant-angle estimation is appropriate for measuring perceived depth. (2) The means of perceived depth measured in the $\mathrm{G}$ condition will be compared with the corresponding means of the NG condition to show that the deceptive distance cues provided by the glasses were effective and that there was a sizable difference in perceived depth in the two conditions. (3) Mean perceived depth obtained in the GP condition, where

Table 1

Mean Tangents and Difference Scores of of Slant Estimates Obtained Under Three Viewing Conditions

\begin{tabular}{ccccccccc}
\hline \multirow{2}{*}{$\begin{array}{c}\text { Dis- } \\
\text { tance }\end{array}$} & NG & & G & Mean & $\mathrm{t}(11)$ & GP & Mean & $\mathrm{t}(11)$ \\
\cline { 4 - 7 } & 2.29 & 1.52 & .77 & 2.45 & 2.03 & .52 & 2.00 \\
80 & 2.13 & 1.22 & .91 & 7.07 & 2.23 & 1.01 & 2.85 \\
\hline
\end{tabular}

Note-Distance is given in centimeters. 
perspective distance cues were added to the effect of the glasses, will be compared with the results of the $\mathrm{G}$ condition. This comparison will show that the perspective distance cues partly couriteracted the effect of the glasses, shifting perceived depth toward the depth measured without the glasses and thus making it more veridical.

Slant angles do not vary linearly with depth. Therefore we changed the slant angle estimates we obtained from our subjects into tangents, which do, and treated the tangents as raw scores for all computations. Since the tangents of the slant angle estimates are relative measures of perceived depth, we are presenting most of our results in these terms.

Preliminary inspection of the results of the G condition showed that the glasses had a significant effect only when a single rod pair was shown, and that the effect of the glasses was much diminished when a presentation at the $40-\mathrm{cm}$ distance immediately preceded a presentation at the $80-\mathrm{cm}$ distance. It seems that the difference between the image sizes of the rod pairs in the near and far positions served as a potent cue to the doubled distance of the rod pair at $80 \mathrm{~cm}$ and overcame the effect of the glasses. Therefore, we limited our comparisons to data obtained in the first successive presentation in each condition. The mean depth estimates (tangents) presented in Table 1 are derived from these first pairs of successive presentations.

(1) To compare the slant angle estimates made without glasses with the actual slant angles, we transformed the mean tangents of the slant estimates obtained in the NG condition, as listed in the first column of Table 1 , back into slant angles. The results of 66.4 and $64.8 \mathrm{deg}$ are in fair agreement with the actual slant angle of our rod pairs, which was $60 \mathrm{deg}$.

(2) A comparison between mean perceived depth in the $G$ and NG conditions, listed in the first and second columns in Table 1 , showed that the glasses were, indeed, effective. As the glasses changed registered distance from 40 to $25 \mathrm{~cm}$, that is, by a factor of $25 / 40$, or .625 , mean perceived depth changed in nearly the same proportion, from 2.29 to 1.52 , by a factor of .664 . At the other location, the glasses changed registered distance from 80 to $36.4 \mathrm{~cm}$, by a factor of .455 . The change in mean perceived depth was not quite as great. It changed from 2.13 to 1.22 , by a factor of .573 . The change in depth at the $40-\mathrm{cm}$ distance was significant at the .02 level, and at the $80-\mathrm{cm}$ distance, at the .001 level.

(3) The main purpose of the experiment, which was to show that linear perspective can operate as a distance cue in stereoscopic depth constancy, was achieved by comparing the results of the GP condition with those of the $\mathrm{G}$ condition. In the GP conditions, where perspective provided veridical distance cues that might counteract the oculomotor cues for dimin- ished distances caused by the glasses, mean perceived depth, as listed in the GP column of Table 1, was significantly higher than in the $G$ condition. This change was significant at the .025 level for perceived depth at the $40-\mathrm{cm}$ distance, and at the .01 level for the 80-cm distance.

Among the 12 subjects who took part in this experiment, there were 6 who each gave his or her very first slant estimate in the $G$ condition at $80 \mathrm{~cm}$ and 6 different subjects who each gave his or her initial slant estimate in the GP condition, also at $80 \mathrm{~cm}$. For these two groups, mean perceived depth was significantly different and greater in the GP condition also. The mean tangent-of-slant estimates for the GP group was 1.98 , and for the $\mathrm{G}$ group it was 1.21 . The difference between these means was significant $[\mathrm{t}(10)$ $=2.22, \mathrm{p}<.05$ ].

As the mean difference between tangents listed in the GP-G column in Table 1 shows, the effect of perspective cues in overcoming the effect of the oculomotor cues was stronger at the $80-\mathrm{cm}$ distance. This was probably due to the larger expanse of the platform pattern visible in front of the rod pair at the 80-cm distance.

\section{SUMMARY}

We have demonstrated that the image sizes of familiar objects as well as linear perspective can operate as distance cues in stereoscopic depth constancy. Because, in Experiment 2, we employed accommodation and convergence deceptively, we also obtained strong evidence of the effectiveness of these oculomotor cues. This finding agrees with the results of Wallach and Zuckerman (1963), who used accommodation and convergence to demonstrate the existence of stereoscopic depth constancy, also employing the oculomotor cues deceptively.

We employed two methods of measuring perceived depth, a direct method, where subjects gave depth estimates, and an indirect one, in which they gave slant estimates that could be transformed into depth estimates. A third method has sometimes been used to measure depth, one in which one marker is placed by the subject so that it appears in the same plane with the near point of a depth interval and another marker is placed so that it appears at the same distance as the far point. This method measures two locations in depth and is therefore not suited to making measurements in connection with stereoscopic depth constancy, which deals with apparent magnitudes of depth intervals.

\section{REFERENCES}

Graham, C. H. Visual perception. In S. S. Stevens (Ed.), Handbook of experimental psychology. New York: Wiley, 1951. 
Ono, H., \& Comerford, J. Stereoscopic depth constancy. In W. Epstein (Ed.), Stability and constancy in visual perception. New York, N.Y: Wiley-Interscience Publication, 1977.

Wallach, H., \& BaCon, J. Two forms of retinal disparity. Perception \& Psychophysics, 1976, 19, 375-382.

Wallach, H., \& FreY, K. J. Adaptation in distance perception based on oculomotor cues. Perception \& Psychophysics, 1972, $11,77-83$.

Wallach, H., Frey, K. J., \& Bode, K. A. The nature of adaptation in distance perception based on oculomotor cues. Perception \& Psychophysics, 1972, 11, 110-116.

Wallach, H., \& Zuckerman, C. The constancy of stereoscopic depth. The American Journal of Psychology, 1963, 76, 404-412.

\section{NOTES}

1. For two different derivations, see Graham (1951) and Wallach and Zuckerman (1963)

2. This experiment is a slightly altered version of one designed by Carl Zuckerman and briefly described in Wallach and Zuckerman (1963). Zuckerman caused convergence to be given deceptively by the use of a pseudoscope. By now, the spectacles developed by Wallach and Frey (1972) have become available for the same purpose. They offer the advantage of altering accommodation along with convergence.

(Received for publication May 29, 1979; revision accepted December 12, 1979.) 